\title{
Turing patterns in a fiber laser with a nested microresonator: Robust and controllable microcomb generation
}

\author{
Hualong Bao,,${ }^{1,}$ Luana Olivieri, ${ }^{1}$ Maxwell Rowley $\odot,{ }^{1}$ Sai T. Chu $\odot,{ }^{2}$ Brent E. Little, ${ }^{3}$ Roberto Morandotti $\odot, 4,5,6$ \\ David J. Moss $\odot,{ }^{7}$ Juan Sebastian Totero Gongora $\odot,{ }^{1}$ Marco Peccianti, ${ }^{1}$ and Alessia Pasquazi $\odot^{1, \dagger}$ \\ ${ }^{1}$ Department of Physics and Astronomy, Emergent Photonics (EPic) Laboratory, University of Sussex, Falmer, \\ Brighton BN1 9QH, United Kingdom \\ ${ }^{2}$ Department of Physics, City University of Hong Kong, Kowloon Tong, Hong Kong SAR, China \\ ${ }^{3}$ State Key Laboratory of Transient Optics and Photonics, Xi'an Institute of Optics and Precision Mechanics, CAS, Xi'an, China \\ ${ }^{4}$ INRS-EMT, 1650 Boulevard Lionel-Boulet, Varennes, Québec, Canada J3X $1 S 2$ \\ ${ }^{5}$ Institute of Fundamental and Frontier Sciences, University of Electronic Science and Technology of China, Chengdu 610054, China \\ ${ }^{6}$ ITMO University, St. Petersburg 199034, Russia \\ ${ }^{7}$ Centre for Microphotonics, Swinburne University of Technology, Hawthorn, Victoria VIC 3122, Australia
}

(Received 27 September 2019; accepted 12 May 2020; published 24 June 2020)

\begin{abstract}
Microcombs based on Turing patterns have been extensively studied in configurations that can be modeled by the Lugiato-Lefever equation. Typically, such schemes are implemented experimentally by resonant coupling of a continuous wave laser to a Kerr microcavity in order to generate highly coherent and robust waves. Here, we study the formation of such patterns in a system composed of a microresonator nested in an amplifying laser cavity, a scheme recently used to demonstrate laser cavity solitons with high optical efficiency and easy repetition rate control. Utilizing this concept, we study different regimes of Turing patterns, unveiling their formation dynamics and demonstrating their controllability and robustness. By conducting a comprehensive modulational instability study with a mean-field model of the system, we explain the pattern formation in terms of its evolution from background noise, paving the way towards complete self-starting operation. Our theoretical and experimental paper provides a clear pathway for repetition rate control of these waves over both fine (Megahertz) and large (Gigahertz) scales, featuring a fractional frequency nonuniformity better than $7 \times 10^{-14}$ with a 100 -ms time gate and without the need for active stabilization.
\end{abstract}

DOI: 10.1103/PhysRevResearch.2.023395

\section{INTRODUCTION}

The formation of patterns in nonlinear dissipative systems is ubiquitous in nature [1]. The emergence of such selforganized periodic patterns on top of a homogeneous background, which was pioneered by Turing, has been observed and investigated in many realms of science, including chemistry, biology, and statistical mechanics [1,2].

With regard to optics, patterns in nonlinear dissipative systems have been studied in both the spatial and the temporal domains [3-15]. The temporal study of such patterns in bistable optical systems [10-15] has received increasing attention in the past decade, in part due to the strong drive to develop optical frequency combs based on microcavities [16-26], referred to as "microcombs." Typical implementations of such microcomb sources involve externally driving a nonlinear Kerr cavity with a continuous wave (cw) laser. This scheme has also emerged as an interesting scenario to study

\footnotetext{
*Corresponding author: H.Bao@ suda.edu.cn

†Corresponding author: a.pasquazi@sussex.ac.uk

Published by the American Physical Society under the terms of the Creative Commons Attribution 4.0 International license. Further distribution of this work must maintain attribution to the author(s) and the published article's title, journal citation, and DOI.
}

nonlinear systems far from equilibrium [27] where localized states, such as cavity solitons, or the modulational instability (MI) of homogeneous states, can appear [19]. This configuration has been extensively studied theoretically and can be efficiently modeled with the Lugiato-Lefever equation [3,28].

Bistable systems, in general, sustain different types of waveforms [1]. Of particular interest for microcombs are localized pulses, namely, solitons, as well as periodic waveforms, usually referred to as patterns, Turing rolls, or cnoidal waves. Solitons are localized waves that can appear as either single or multiple pulses. Because these pulses are highly confined in time, solitons can achieve broad and smooth spectra and have, as such, been of significant interest to the microcomb community for applications in spectroscopy, optical ranging, and communications [29-38]. On the other hand, patterns are nonlocalized waves which, in contrast to solitons, are periodic within the cavity space and, in general, achieve fewer optical frequency modes. Nonetheless, the intrinsic nature of periodic waves leads to patterns that exhibit a more robust type of phase locking and, for this reason, they are better suited to applications that require highquality mode locking of a high repetition rate source, such as pure microwave, terahertz generation, or low-noise ultrafast telecommunications [38-41].

Turing rolls can appear in Lugiato-Lefever systems that are pumped in either the normal or the anomalous dispersion 

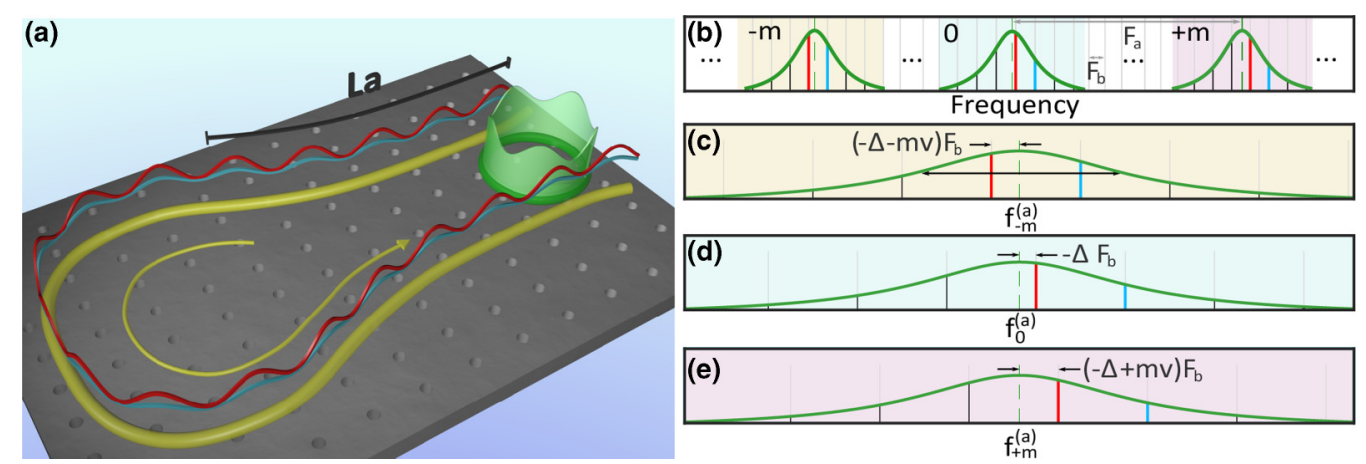

FIG. 1. (a) Depiction of the laser operation. The Kerr microresonator (green ring) is nested into an amplifying fiber loop (yellow). A Turing pattern waveform (light green) is excited in the microcavity and is sustained by a leading-order (red) and a first-order (blue) "supermode" waveforms from the amplifying loop. These supermodes are periodical with the microcavity length, highlighted by a line segment $L_{\mathrm{a}}$. (b) Spectral distribution of the modes in the cold-cavity condition. The microcavity resonances (green) have a FSR denoted $F_{\mathrm{a}}$ whereas the FSR of the amplifying main-cavity modes (black) is denoted $F_{\mathrm{b}}$. The leading-order and first-order supermodes are plotted in red and blue, respectively. (c)-(e) Zoom of the $m$ th, central, and $-m$ th resonances. $\Delta$ is the normalized frequency offset between the central frequency of the leading supermode and the microcavity resonance. $v$ is the normalized FSR detuning, appearing when the two cavities are not commensurate.

regime $[1,3,17,42]$. These different cases have an effect on the transition from Turing patterns to higher complex nonlinear regimes and/or to spatiotemporal chaos. Anomalous dispersion configurations, for instance, are known for being prone to a limited regime of stability for Turing patterns. The transition to spatiotemporal chaos has been recently studied in the monostable regime of the Lugiato-Lefever equation, highlighting the evolution of the state into a turbulent regime [43]. The transition to chaos, in general, is what limits the maximum conversion efficiency of the system. In the normal dispersion regime, local mode hybridization has been implemented to enhance the pump conversion efficiency by increasing the region of existence of the Turing patterns, thus, avoiding the emergence of subcombs [40]. This technique has demonstrated combs with a fractional frequency nonuniformity measured at $7.3 \times 10^{-14}$ with a 1 -s time gate and allows one to design a sample that produces an oscillator with a single well-defined repetition-rate.

Looking more generally at alternative approaches for microcomb generation, we introduced a scheme based on nesting a high- $Q$ nonlinear microcavity into an amplifying fiber loop [44-46]. Such a scheme allows for coherent pulsed states $[44,46]$ and as recently demonstrated sustains a class of temporal cavity solitons, namely, laser cavity solitons [47]. Microcomb laser cavity solitons are intrinsically backgroundfree bright solitons with a mode efficiency exceeding $96 \%$, compared to the theoretical limit of 5\% for bright LugiatoLefever solitons. Remarkably, these solitons are reconfigurable in terms of repetition rate by simply acting on the cavity length of the fiber loop.

Here, we focus our attention on a different set of solutions of this laser: nonlocalized Turing pattern waves. Revisiting our original results for stable pulsed solutions [44] and implementing a comprehensive mean-field model, in conjunction with our measurements, we explain the generation dynamics of these waves via a MI process. We examine the range of parameters for which it is possible to select the repetition rate for some multiple of the microcavity free-spectral range (FSR) [48] and show that it can, in fact, be continuously tuned by up to $10 \mathrm{MHz}$. Finally, we demonstrate extremely robust and high-quality mode locking, obtaining a frequency variation in the repetition rate well below the hertz level, making such patterns particularly attractive for the generation of ultrastable microwave sources. Our approach achieves a deviation in the repetition rate less than $7 \times 10^{-14}$ for time gates of $100 \mathrm{~ms}$ with the ability to tune the repetition rate by adjusting a simple parameter (or component, i.e., a delay line). We verify experimentally continuous tuning of up to $10 \mathrm{MHz}$ and Turing pattern generation with repetition rate of approximately 100, 150, and $200 \mathrm{GHz}$.

\section{THEORETICAL BACKGROUND}

We begin by describing the two traveling-wave resonator system as depicted in Fig. 1(a), which features a Kerr cavity, such as a microring (green ring), nested in a main amplifying cavity, e.g., a gain fiber loop (yellow loop). Their roundtrip time and length, expressed in standard units, are $T_{(a, b)}$ and $L_{(a, b)}$ with FSRs $F_{(a, b)}=T_{(a, b)}^{-1}$, respectively. We consider $T_{b} \gg T_{a}$ and define an integer $M$ such that

$$
F_{a}=(M-v) F_{b} .
$$

The relative cavity-period mismatch $|v|<\frac{1}{2}$ that allows for modeling noncommensurate loops is of particular importance. As we will see below, the FSR mismatch between the two cavities regulates the region of existence of solitons and patterns. In the latter case, we will show that $v$ plays a fundamental role also in defining the properties of the MI of a constant stationary state, which defines the typical features of pattern formation and their FSRs.

We define the spatial coordinates $X_{(a, b)}$ and assume that the optical fields in the two cavities $A\left(T, X_{a}\right)$ and $B\left(T, X_{b}\right)$, in square root of Watts are slowly varying in time $T$ in seconds. It is important to derive an expression for the field in the main cavity that permits an easy manipulation in terms of supermodes also determining the existence and stability of several different stationary states in the system. A supermode is a wave formed by a set of equally spaced modes of the main cavity, the relative spacing of which is given by the microcavity FSR $F_{a}$. This concept is depicted in 
Figs. 1(b)-1(e). It is useful, then, to first expand the field $B\left(T, X_{b}\right)$ within the fiber cavity in the set of cavity modes $b_{n}(T)$,

$$
B\left(T, X_{b}\right)=\sum_{n=-\infty}^{\infty} b_{n}(T) \exp \left[2 \pi i n \frac{X_{b}}{L_{b}}\right] .
$$

The frequency of these modes, spaced by the main-cavity FSR $F_{b}$, is depicted by means of the gray lines in Fig. 1(b). We can now group together all the modes spaced by the microcavity FSR, which, as expressed by Eq. (1), is approximately $M$ times larger than the main-cavity FSR. To this aim, we express the index $n$ of the set $b_{n}$ as

$$
n=m M+q,
$$

where the integer $m$ can take an arbitrary value whereas the integer $q$ is given such that $|q|<M$ and defines the order of the supermode. Figure 1(d) visually depicts those modes in red for the leading-order mode with $q=0$ and in blue for the first-order mode $q=1$. We can now focus our attention on the modes $b_{n}=b_{m M+q}$ featuring the same $q$. These modes are Fourier transformed in the space $X_{a}$ of the microcavity,

$$
B_{q}\left(T, X_{a}\right)=\sum_{m=-\infty}^{\infty} b_{m M+q}(T) \exp \left[2 \pi i m \frac{X_{a}}{L_{a}}\right] \text {. }
$$

Here the supermode with $q=0$ has the best spectral overlap with the microcavity resonances. The fields $B_{q}$ in the direct space are summarized in Fig. 1(a) where the line segment $L_{a}$ covers a length $X_{a}$ equal to the microcavity round-trip.

As we have demonstrated [47], the two-cavity system can be represented by a set of mean-field equations in terms of the field in the microcavity $A$, and a total number of $N$ supermode fields $B_{q}$ in the main cavity (where $N \ll M$ ). As such, we consider the following parameter definitions: the waveguide first- and second-order dispersion $\beta_{(a, b)}^{(1,2)}$, in $\mathrm{s} \mathrm{m}^{-1}$ and $\mathrm{s}^{2} \mathrm{~m}^{-1}$, respectively; the amplifying gain within the main-cavity $G$, in $\mathrm{m}^{-1}$; the corresponding bandwidth $\Delta F_{F}$, dictated by a bandpass filter, in hertz; the Kerr waveguide coefficient $\gamma$, in $\mathrm{W}^{-1} \mathrm{~m}^{-1}$ [49]; the $-3-\mathrm{dB}$ linewidth of the microcavity resonance $\Delta F_{A}$ in hertz, which is directly related to the coupling coefficient of the two cavities $\theta=\pi \Delta F_{A} T_{a}$ [50]. Furthermore, $\Delta$ is the cavity-frequency offset, normalized with $F_{b}$. The dimensionless system reads

$$
\begin{aligned}
\partial_{t} a & =\frac{i \zeta_{a}}{2} \partial_{x x} a+i|a|^{2} a-\kappa a+\sqrt{\kappa} \sum_{q=-N}^{N} b_{q} \\
\partial_{t} b_{q}= & -v \partial_{x} b_{q}+\left(\frac{i \zeta_{b}}{2}+\sigma\right) \partial_{x x} b_{q} \\
& +2 \pi i(\Delta-q) b_{q}+g b_{q}-\sum_{p=-N}^{N} b_{p}+\sqrt{\kappa} a
\end{aligned}
$$

Here, the equations have been normalized as in Ref. [47]. Specifically, we make the normalization of the propagating time against the main-cavity period in the moving pulses frame $t=T T_{b}^{-1}$, whereas the fast cavity time, defined as $x=X_{a} L_{a}^{-1}-T T_{a}^{-1}$, is normalized against the microcavity roundtrip. Further normalization of the system properties are for the microcavity field $a=A \sqrt{\gamma T_{b} L_{a} T_{a}^{-1}}$, the main-cavity field $b_{q}=B_{q} T_{b} T_{a}^{-1} \sqrt{\gamma L_{a}}$, the amplifying gain $g=G L_{b}$, the normalized cavity dispersions $\zeta_{a}=-\beta_{a}^{(2)} L_{a} T_{b} T_{a}^{-3}, \zeta_{b}=$ $-\beta_{b}^{(2)} L_{b} T_{a}^{-2}$, and the filtered main-cavity bandwidth $\sigma=$ $\left(2 \pi \Delta F_{F} T_{a}\right)^{-2}$. The normalized coupling between the two cavities is defined such that $\kappa=\theta T_{b} F_{a}=\pi \Delta F_{A} T_{b}$, which directly provides the number $\kappa \pi^{-1}$ of main-cavity modes per microring resonance. Similar equations have also been studied for coupled waveguide laser configurations [51-53] and frequency selective feedback lasers [54].

It is important to stress that the system does not involve any form of fast gain saturation [12], which is not necessary for sustaining the types of waves studied here. We note, however, that slow saturation of the lasing material (in ytterbium erbium-doped fiber this value is on the order of $10 \mathrm{~ms}$ [55]) does play a role in setting the optical field energy within the system. The analysis reported here, then, is focused on explaining the nature of the stationary states and the ultrafast wave dynamics, such as MI, which are instrumental in defining the different types of stable states.

Since patterns may arise from the instability of a cw solution, it is useful to find the homogeneous solutions, $a(t)=$ $\sqrt{I} \exp [-2 \pi i \phi t]$ and $b_{q}(t)=\bar{b}_{q} \exp [-2 \pi i \phi t]$, where $I$ is the constant intensity of the microcavity field, $\bar{b}_{q}$ is the constant field for the supermode $q$, and $\phi$ is the normalized frequency of the stationary state. Among this class of solutions, the system also admits the trivial solution $a(t)=0$ and $b_{q}(t)=0$.

For $N>0$, we find $2 N+1$ states which are approximated by the formulas,

$$
\begin{gathered}
\phi_{ \pm}^{(q)}=-\left[\Delta-q \pm(2 \pi)^{-1} \sqrt{(1-g) g}\right] \\
I_{ \pm}^{(q)}=\left(2 \pi(\Delta-q) \pm \frac{\sqrt{g}(1-g+\kappa)}{\sqrt{1-g}}\right)
\end{gathered}
$$

with $q$ spanning from $-N$ to $N$ and the field in the microresonator resulting in $a_{ \pm}^{(q)}(t)=\sqrt{I_{ \pm}^{(q)}} \exp \left[-2 \pi i \phi_{ \pm}^{(q)} t\right]$. These formulas are exact for $N=0$ and allow us to identify the different states, which can be readily refined by numerical integration. Note that the $I_{ \pm}^{(q)}$ states have most of their energy contained within the supermode of order $q$ with $b_{q, \pm}^{(q)} \approx$ $\sqrt{\kappa I_{ \pm}^{(q)}}\left[1 \mp i \sqrt{g(1-g)^{-1}}\right] \exp \left[-2 \pi i \phi_{ \pm}^{(q)} t\right]$ and $b_{p, \pm}^{(q)} \approx 0$ for $p \neq q$.

\section{Modulational instability of the homogeneous, stationary states}

Equations (5) and (6) define a homogeneous system which is solved by the trivial state solutions $a=0$ and $b_{q}=0$. The MI of such states provides important information on the region of existence of localized and nonlocalized solutions as discussed in Ref. [47] for the formation of solitons. Here, we mostly focus our attention towards nonlocalized solutions, which can arise from the MI of the zero-energy background. The MI gain of the system is obtained by calculating the real part of the eigenvalues associated with the linear perturbation of the stationary state [1]. Such perturbation is a monochromatic wave with frequency $f$. We will refer to this frequency as the dynamical or perturbation frequency.

Figure 2 summarizes some significant results from the MI analysis as a function of the detuning parameter $\Delta$. Since the 

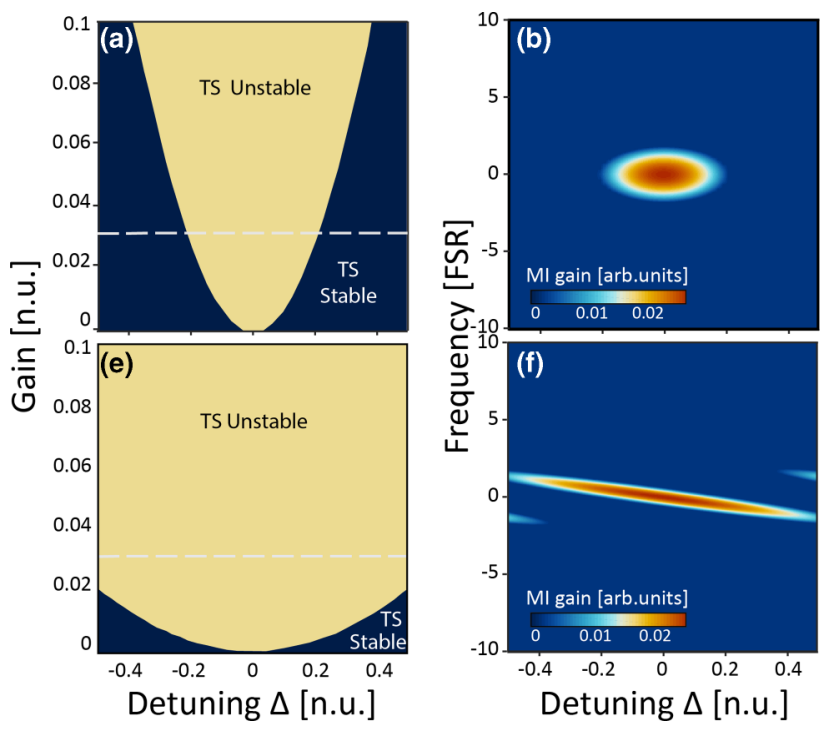
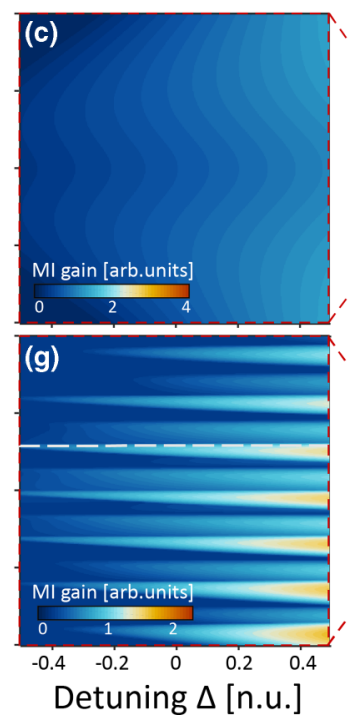

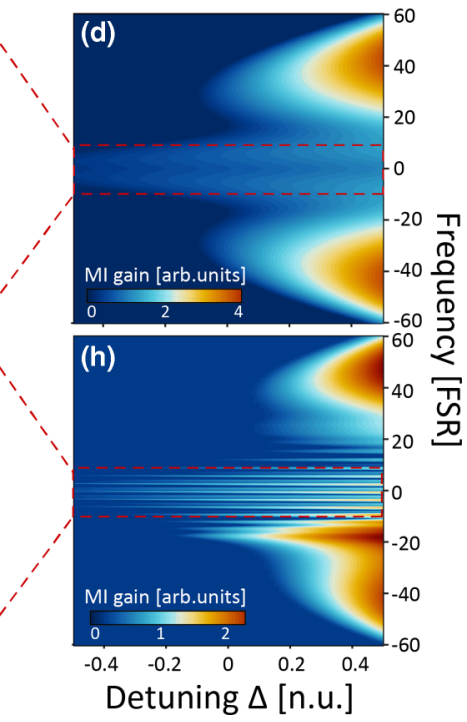

FIG. 2. MI maps for the stationary states. For the calculations: $\zeta_{a}=1.7 \times 10^{-4}, \quad \zeta_{b}=3.5 \times 10^{-4}, \sigma=2.5 \times 10^{-4}$, and $\sqrt{\kappa}=2.5$, which are compatible with the experimental regime discussed in the sections below. Here, we used $N=7$, corresponding to a total of 15 supermodes. (a) MI map for the trivial solution (TS) showing the regions of instability as a function of the cavity gain $g$ and detuning $\Delta$ with $v=0$. (b) MI gain of the zero solution with $g=0.06$ (indicated by a white dashed line) and $v=0$. (c) MI gain of the low-energy cw state $I_{0}^{+}$. (d) Zoom out of (c). (e)-(h) are the same as (a)-(d) with $v=1 / 3$.

system is periodic with period $2 \pi$, we focus our attention on the base range $-1 / 2<\Delta<1 / 2$. Figures 2(a)-2(d) and 2(e)2 (h) show the results for the matched cavities $(v=0)$ and for cavities with a period mismatch $(v=1 / 3)$, respectively. The maps in Figs. 2(a) and 2(e) generally summarize the stability regions for the trivial state as a function of the detuning $\Delta$ and cavity gain $g$. In the unstable regions (yellow shading) it is implied that the MI gain is positive for, at least, one perturbation frequency. Here, we note that the zero-energy state is always unstable for perfectly matched cavities $(\Delta=0)$. Examining the MI gain over the range of perturbation frequencies [reported in Figs. 2(b) and 2(f)] at a specific value of the cavity gain: $g=0.06$, we note that the maximum MI gain is observed for the perturbation frequency $f=0$, resulting in a growth of a cw perturbation from the trivial state.

It is also important to properly evaluate the MI spectrum for homogeneous nontrivial states. Within the set of parameters studied here, the MI analysis suggests that all $\mathrm{cw}$ states can be unstable within the base range. We focus our attention on state $I_{+}^{(0)}$. As can be verified with Eq. (8), this state represents the lowest-energy $\mathrm{cw}$ solution across the region $\Delta<0$. A plot of the intensities of the cw states as a function of $\Delta$ in the range of parameters studied here is also reported in Fig. 3(c) for better visualization. The MI for the matched cavities $(v=0)$ and for cavities with a period mismatch $(v=$ 1/3) are reported in Figs. 2(c) and 2(g), which cover the lower dynamical frequency range of the complete MI spectrum presented in Figs. 2(d) and 2(h). The latter plots show that this MI gain is very wide for $\Delta>0$ (red-detuned cavities) and extends towards very high frequencies. We recall that this is the region where solitons, which are localized waves, exist [47]. The MI gain for the range $\Delta<0$ (blue-detuned cavities), conversely, can be more limited in bandwidth, hence, it is easier to induce and control nonlocalized patterns.
In more detail, Figs. 2(g) and 2(h) show that the cavityperiod mismatch $v$ can actually induce a set of new "tongues" in the lower-frequency spectrum of the MI gain. This can be understood considering that the presence of a filter within a laser cavity is known to allow the selection of pulsed states at multiples of the free-spectral range associated to the filter cavity by means of the Vernier effect $[48,56]$. In the formalism of this paper, when the mismatch $v$ is the inverse of some integer number $K$ (i.e., $v=1 / K$ ), the frequency of the maincavity mode can align to every $K$ th microring resonance mode. This feature has also been shown, in some cases, to force the oscillation at every $K$ th FSR of the intracavity filter $[48,56]$ and, in fact, stable oscillation has previously been obtained in configurations similar to that of our paper when a nonlinear microcavity was employed as a filter $[46,48]$.

Our analysis, here, allows us to clearly interpret the origin of the set of Turing pattern states in terms of a cascading MI. Indeed, the MI spectrum of the cw states can present a sharp maximum around the dynamic frequency $f=K$. Figure 2(g) shows an example for the case $v=1 / 3$ where the normalized frequency $f=3$ is highlighted with a dashedwhite line. Figures 2(f) and 2(g), then, reveal the existence of a self-starting regime of the Turing pattern state defined for a set of values where $\Delta<0$, an example of which is presented in Fig. 3. Here, the MI gain of the trivial state initially seeds the growth of a cw state, which, once generated within the cavity, will have its dynamics regulated by the MI gain of the homogeneous state. In this case, the sharp peak at $f=3$ induces the formation of a stable Turing pattern at this frequency.

These considerations explain why this set of Turing patterns is very commonly observed [44-46,48]. They are a set of intrinsically self-starting solutions that can be readily reformed if the energy in the cavity is lost through some 

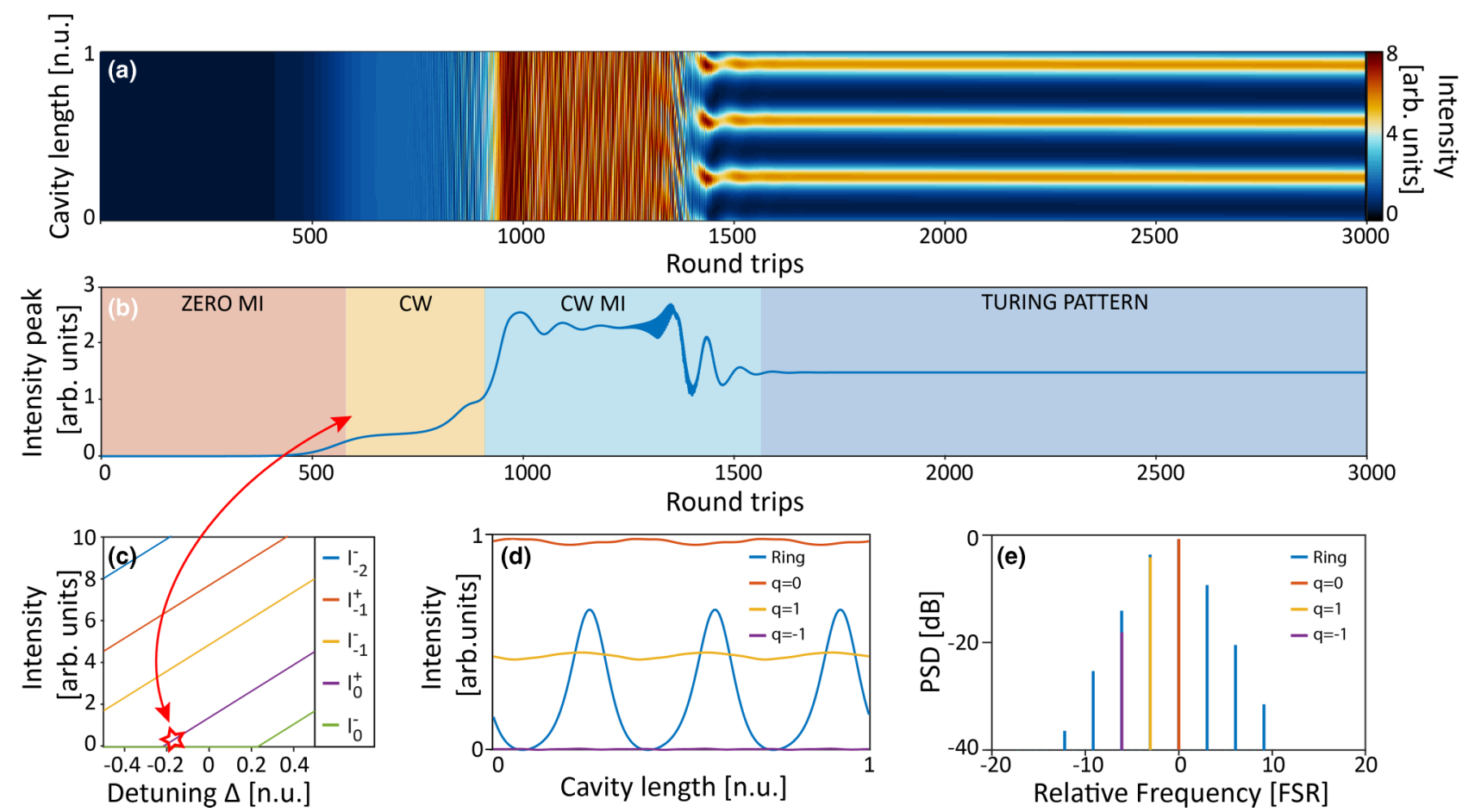

FIG. 3. Self-starting Turing pattern states. The zero solution evolves into state $I_{+}^{(0)}$, which is also unstable and gives rise to a Turing pattern. The simulation parameters are: $\zeta_{a}=1.7 \times 10^{-4}, \zeta_{b}=3.5 \times 10^{-4}, \sigma=2.5 \times 10^{-4}, \Delta=-0.17, \sqrt{\kappa}=2.5, g=0.0365, v=-0.3342$, $N=7$. (a) Evolution of the temporal profile of the field in the microcavity with the $y$ axis normalized to the microcavity length. (b) Maximum intensity of the evolution in the microcavity. (c) Low-energy stationary states. (d) Output time profile of the fields in the micro- and main cavities. (e) Output spectral profiles of the fields within the micro- and the main cavities with power spectral density (PSD) as a function of the normalized relative frequency.

perturbation. The presence of sharply localized tongues in the MI spectrum allows for a very clean selection of the dynamic frequency $f$, which is controlled directly by the cavity mismatch $v$. We will discuss this along with the effect of small changes of the cavity mismatch $v$ in Sec. III where we present also our experiments.

Finally, the strong skew in the MI spectrum induced by the period mismatch $v$ [as shown in Figs. 2(e)-2(h)], shifting the higher frequencies towards the border of the base range of the detuning $\Delta$, may be indicative that the system can support Faraday instabilities, which have been recently studied in resonators with regions of differing group-velocity dispersion $[57,58]$.

\section{EXPERIMENTAL IMPLEMENTATION}

A schematic of our experimental setup is shown in Fig. 4(a). It is composed of a high- $Q\left(>10^{6}\right)$ integrated Hydex microresonator [59], an EYDFA, a free space delay line, a tunable passband filter, and an optical isolator. The microresonator FSR is $\sim 49 \mathrm{GHz}$ with a linewidth of $\sim 100 \mathrm{MHz}$. The EYDFA provides relatively large gain over a short fiber length $(<1.5 \mathrm{~m})$, the gain profile being shaped by the tunable passband filter (6-nm 3-dB bandwidth) centered at $\sim 1550 \mathrm{~nm}$. The free space delay line is used for controlling the phase of the main cavity modes with respect to the microcavity modes. The total main-cavity length is $\sim 3.5 \mathrm{~m}$, resulting in a mode spacing of $\sim 55 \mathrm{MHz}$. The use of fully polarization maintaining fiber components prevents any nonlinear polarization rotation effects [19].

Frequency comb-assisted spectroscopy $[46,47,60]$ is performed in order to extract the exact positions of the oscillating microcomb lines in the hot resonator (i.e., during operation). A scanning $\mathrm{cw}$ laser probes the resonance profiles and oscillating microcomb lines whereas a reference frequency comb (Menlo Systems) and a Mach-Zehnder interferometer (MZI) are used to perform the highly accurate frequency calibrations as illustrated in Fig. 4(b).

A frequency uniformity measurement setup $[40,61,62]$ is used to extract the microcomb repetition rate deviation. Here, three adjacent microcomb lines are selected and beat with three adjacent comb lines of a reference frequency comb. The three beat notes, B1, B2, and B3 are combined (B1-B2 and B2-B3) through two frequency mixers. We retained only the repetition rate frequency signal which was detected by a high-resolution frequency counter in the ratio counting mode $(10-\mathrm{mHz}$ frequency error at $1 \mathrm{~s})$.

The optical spectrum and autocorrelation from the system are monitored with the intracavity optical coupler at the microresonator drop port. In particular, the frequency comb-assisted spectroscopy measurements utilize the small backreflected signal from the input port whereas the frequency uniformity measurements use the microresonator through the port signal. 

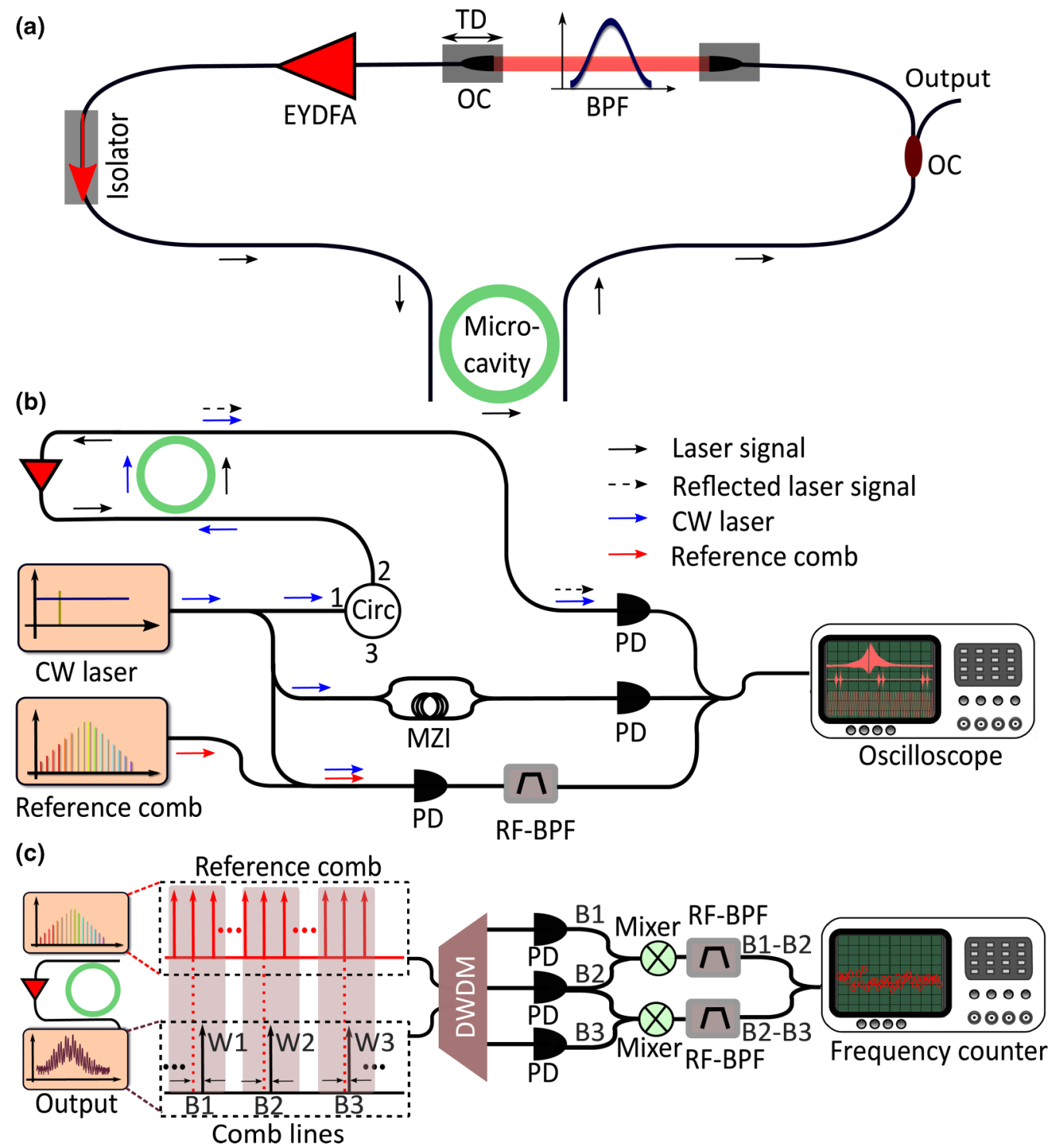

FIG. 4. (a) Experimental setup of the micro-cavity-filtered laser. Erbium-ytterbium-doped fiber amplifier: EYDFA; translation delay stage: TD; bandpass filter: BPF; output coupler: OC. (b) Diagnostic setup for the frequency comb-assisted spectroscopy. Photodiode: PD; MZI; radio-frequency bandpass filter: rf-BPF. (c) Diagnostic setup for the frequency uniformity measurement. The beat notes B1-B3 among the three desired comb lines W1-W3 and their adjacent reference frequency comb lines are selected using a dense wavelength division multiplexer (DWDM). They are then mixed and counted by a high-resolution frequency counter.

\section{A. Turing pattern selection via the Vernier effect}

We first verified the properties of the Turing pattern states controlled by the Vernier effect. As discussed in Fig. 3, the cavity detuning and mismatch parameters, respectively, $\Delta$ and $v$, need to be carefully adjusted in order to access this regime. Experimentally, this control is obtained by acting on the delay line in the fiber cavity whereas the position of the oscillating microcomb lines can be verified by means of the intracavity scanning spectroscopy setup. The experimental results shown in Fig. 5 display a range of cases with comb teeth spaced by two to four FSRs. These measurements are fit using Eqs. (5) and (6) with simulation parameters: $\sqrt{\kappa}=2.5, \sigma=2.5 \times$ $10^{-4}, \zeta_{a}=1.7 \times 10^{-4}, \quad \zeta_{b}=3.5 \times 10^{-4}, N=7$, and values $\left|\beta_{(a)}^{(2)}\right|=20$ and $\left|\beta_{(b)}^{(2)}\right|=60 \mathrm{ps}^{2} / \mathrm{km}$ (within our experimental constraints).
Figures 5(a), 5(d), and 5(g) show the experimental optical spectra as well as autocorrelation traces and the position of the central oscillating comb line (i.e., the mode with the highest power spectral density). The corresponding simulated spectral traces are presented in Figs. 5(b), 5(e), and 5(h) for a set of Turing patterns with frequency spacing at two to four FSR. The high contrast autocorrelation traces are indicative of the waveforms' coherence. The intracavity spectrum shows an oscillating line within the microcavity resonance blue-detuned by $13.9,12.4$, and $22.1 \mathrm{MHz}$. For the numerical fitting in Figs. 5(b), 5(e), and 5(h), settings of $\Delta=-0.36, \Delta=-0.17$, and $\Delta=-0.54$ were used, along with mismatch parameters $v=\frac{1}{2}, v=1 / 3$, and $v=1 / 4$. The analysis around Fig. 2 has indicated that, for these parameters, the MI of the trivial solution leads to the emergence of a homogeneous state. Figures 5(c), 5(f), and 5(i) depict the MI gain spectrum of such a 

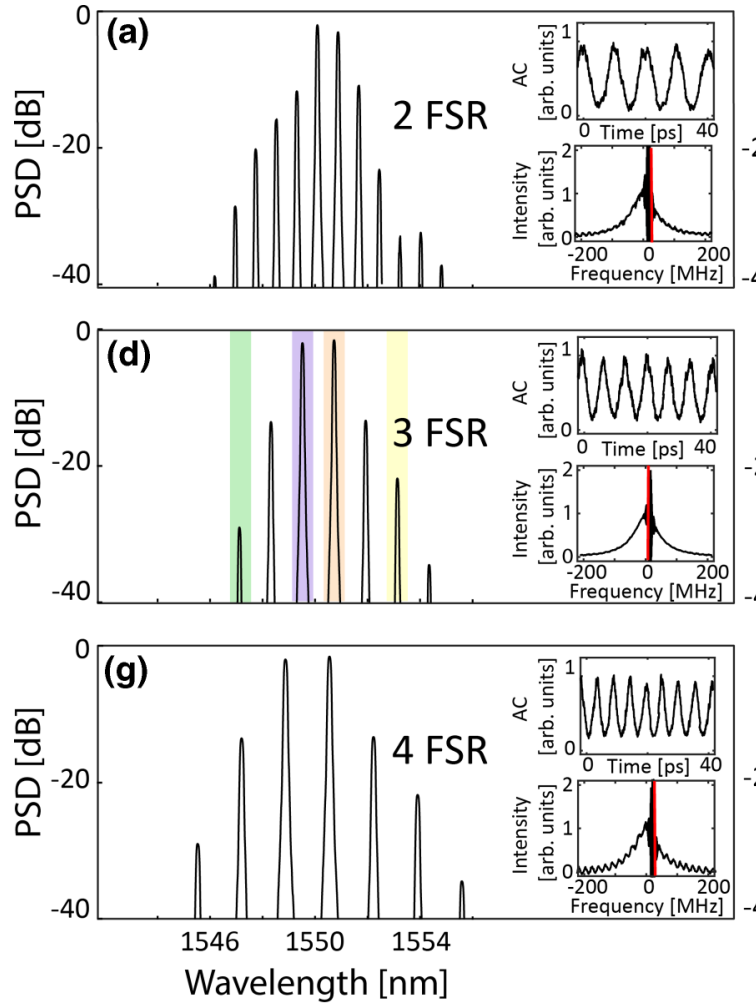
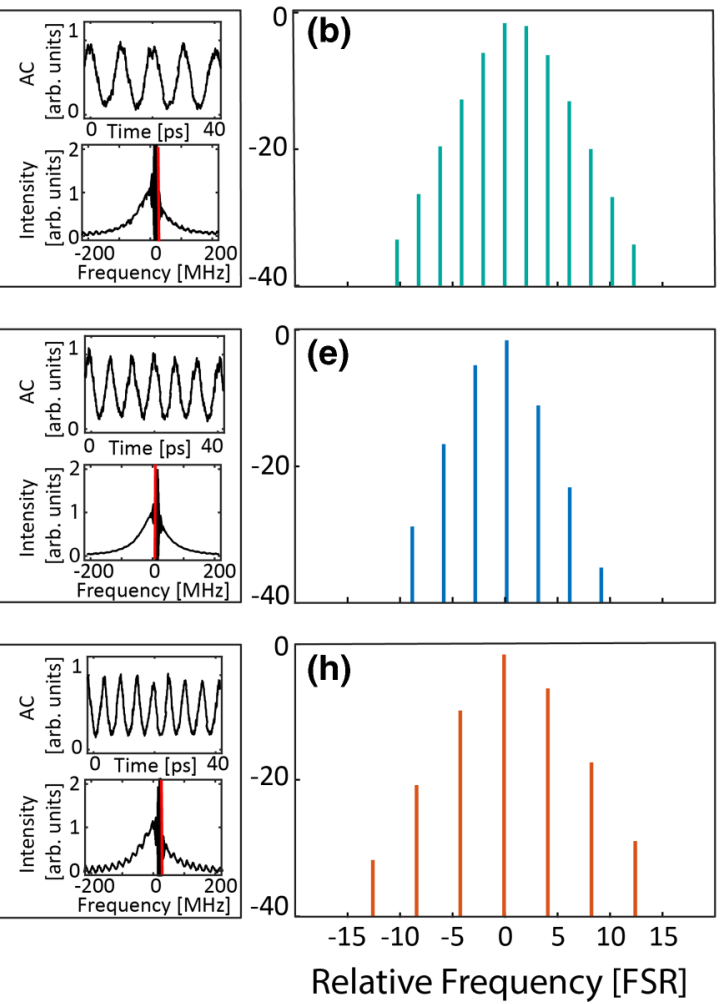
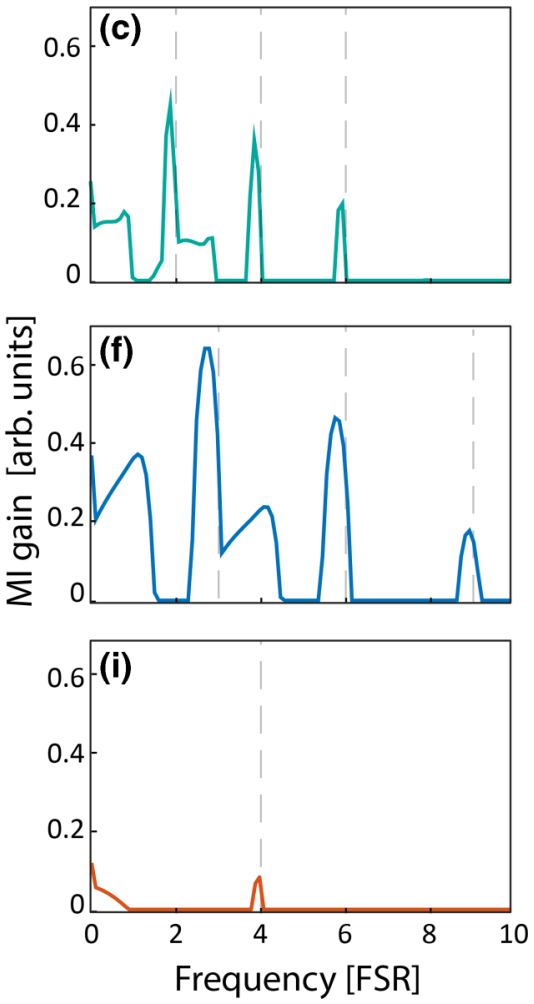

FIG. 5. (a), (d), and (g) Experimental optical power spectral density (PSD) for the microcomb modes. The insets depict the autocorrelation (AC) and the intracavity spectrum of the oscillating spectral mode with the highest power. The red line here shows the position of detuning $\Delta$ used in the respective simulations. The color shadings in (d) are in reference to the resonance profiles presented in Fig. 6. (b), (e), and (h) Simulated optical spectra calculated at $v=1 / 2,1 / 3,1 / 4$, respectively, with corresponding gain settings and cavity detunings of (b) $g=0.0400, \Delta=-0.36$, (e) $g=0.0320, \Delta=-0.17$, and (h) $g=0.0501, \Delta=-0.54$. (c), (f), and (i) MI gain spectrum of the cw states. In the calculations: $\sigma=2.5 \times 10^{-4}, \zeta_{a}=1.7 \times 10^{-4} \zeta_{b}=3.5 \times 10^{-4}, \sqrt{\kappa}=2.5$, and $N=7$

state, given each set of parameters. The related gain spectrum shows a clear maximum around the perturbation frequency at approximately two to four FSR, which is consistent with the experimentally observed comb line spacings.

\section{B. Fine-Tuning and Phase Locking of the Turing patterns}

Experimentally, the fine tunability of the repetition rate can be obtained by slight modification of the main-cavity length via a tunable delay line. Stable laser oscillation is observed throughout the whole tuning process, highlighting the capability of the system to maintain this state. We present an example of this tunability, with intracavity spectroscopy measurements of a Turing pattern with a period equal to three times the FSR of the nested microresonator. Similar tunability has been verified on all the cases presented above. We use five different fiber cavity lengths covering a range of $400 \mu \mathrm{m}$. The intracavity spectroscopy measurements allow us to visualize the shift of the resonating lines within the microcavity resonances and is summarized in Fig. 6. Note that, in all these cases, we observe that the line with the highest power spectral density (highlighted with orange shading) remains in almost the same position with respect to the central resonance (the variations are within a few megahertz), whereas sideband modes experience much larger shifts which are indicative of a change in the repetition rate. From these lines, we extract a normalized detuning parameter $\Delta=-0.35$ for all the cases, whereas the mismatch parameter $v$ for the respective maincavity lengths varies by $0.044,0.064,0.076,0.092$, and 0.106 around the value of $v=1 / 3$.

Figure 7 shows further simulations that agree well with the experimental observations on the tunability of the repetition rate. The numerical parameters are the same as in previous simulations shown in Fig. 3 where the mismatch $v$ was changed from -0.33 to -0.39 : corresponding to an experimental change in cavity length of $400 \mu \mathrm{m}$. The stability that is maintained over this range of the mismatch parameter in our simulations emphasizes the strong agreement between our theoretical model and experiments. The change in group velocity of the pattern obtained in Fig. 7, compared to Fig. 3, indicates the fine-tuning of the repetition rate: this is visible from the clear inclination of the patterns in Fig. 7(a), which is better shown in the inset of the figure. These simulations also confirm the strong phase locking of the Turing pattern states as already discussed within the context of the Lugiato-Lefever comb [39]. To highlight this, the differential phase evolution of the absolute phase for three of the modes is presented in Fig. 7(d), demonstrating the strong phase locking of the repetition rate of the state.

For a more accurate measurement of the repetition rate detuning and stability, we employ a frequency uniformity measurement technique as illustrated in Fig. 4(c) $[40,61,62]$ to probe the equidistance of the generated comb lines. The recorded mixed frequencies for the experiment in Fig. 6 are 

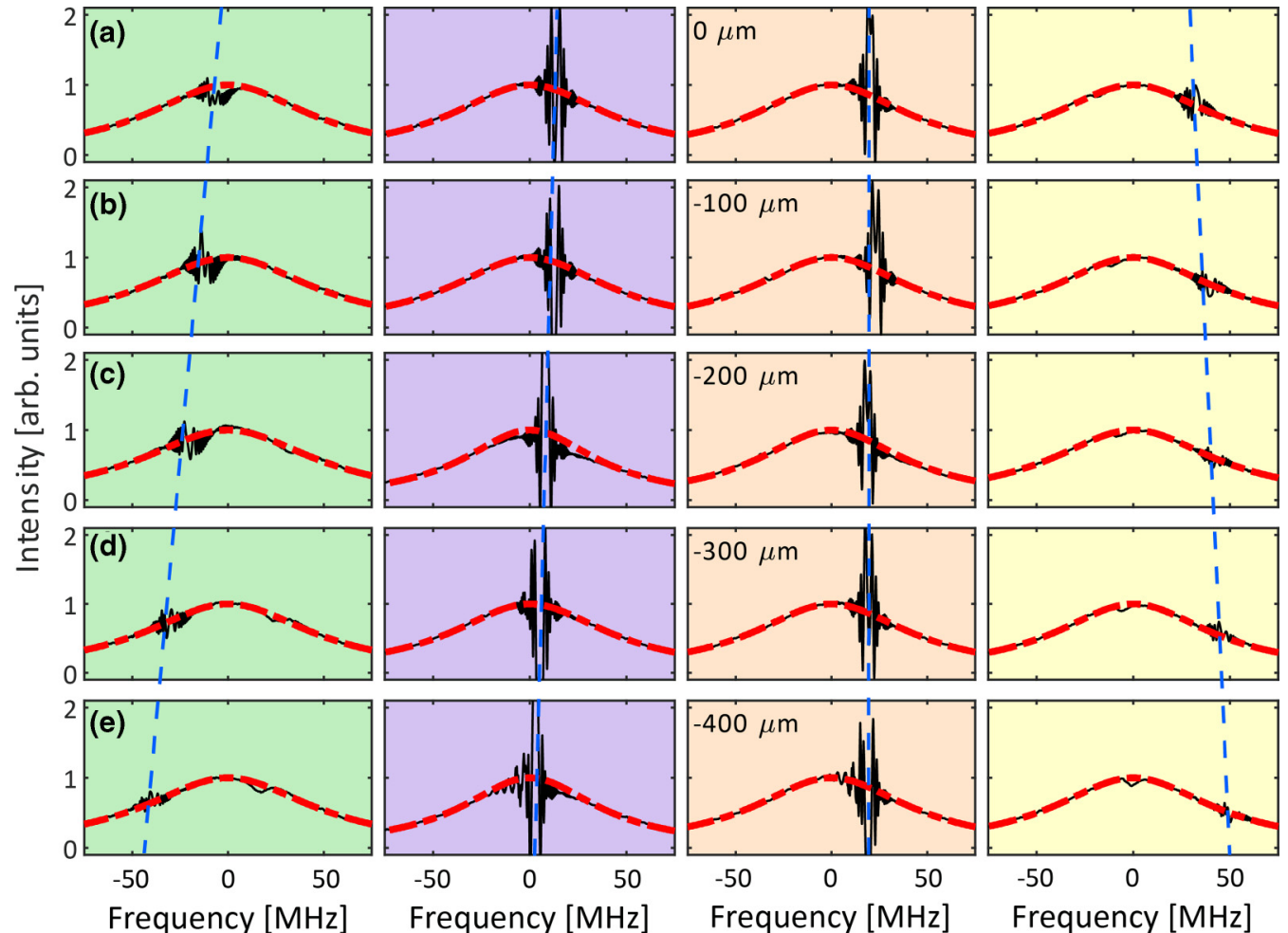

FIG. 6. Experimental resonance profiles and beat note signals obtained with the probe laser scanning oscillating comb lines. (a)-(e) Descending rows correspond to a relative change in the main cavity length of $0,-100,-200,-300$, and $-400 \mu \mathrm{m}$. The four sequential color-shaded plots correspond to different comb line wavelengths, 1547.13, 1549.47, 1550.64, and $1553.01 \mathrm{~nm}$, respectively, from the left to the right panels as indicated by the color shading in Fig. 5(d). The dashed red line is a Lorentzian fit to the profile of the hot resonance whereas the vertical dashed-blue lines indicate the relative shift of the oscillating comb lines within their respective resonances.
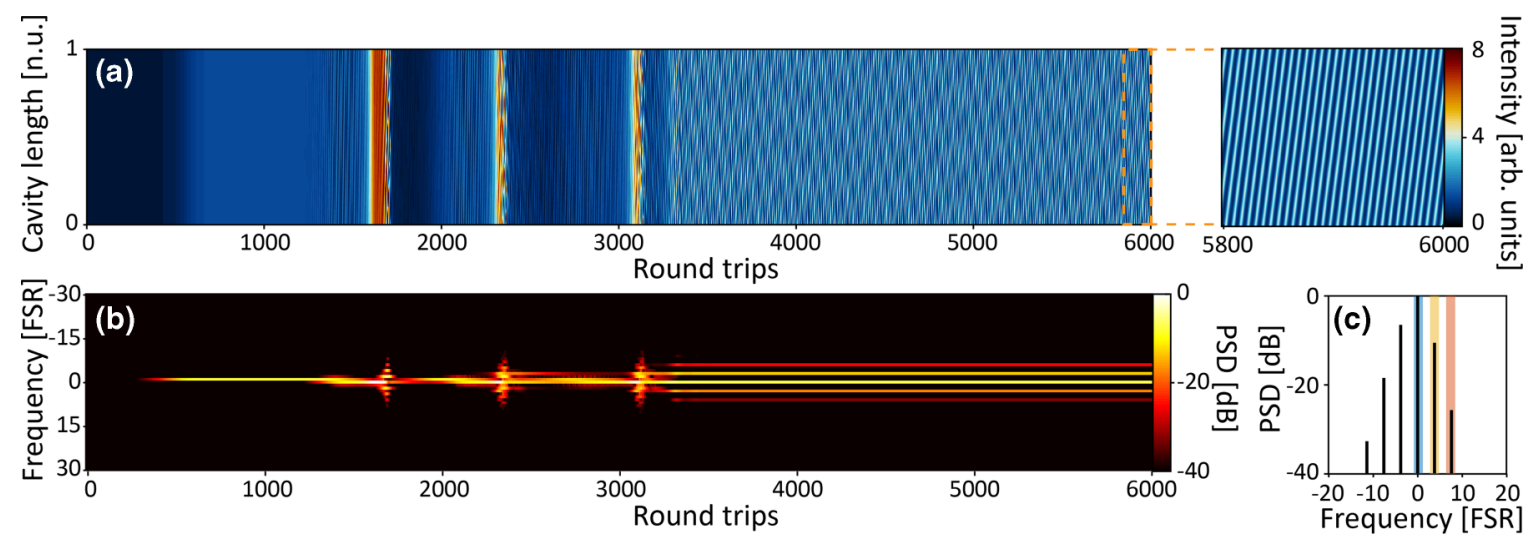

(d)

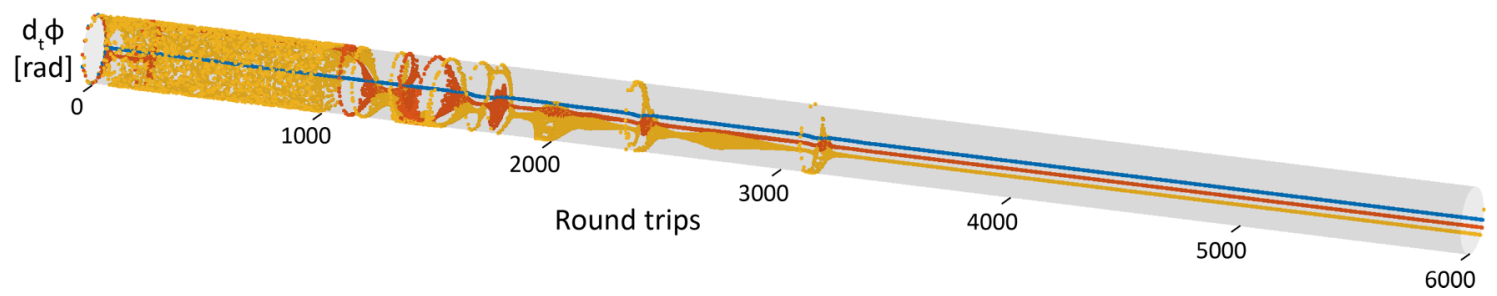

FIG. 7. (a) and (b) Numerical results showing the evolution of the (a) temporal and the (b) spectral profile of the field. The temporal evolution has a corresponding zoom of the stable operation to illustrate more clearly the pattern. For the simulations: $\zeta_{a}=1.7 \times 10^{-4}$, $\zeta_{b}=3.5 \times 10^{-4}, \sigma=2.5 \times 10^{-4}, \Delta=-0.17, \sqrt{\kappa}=2.5, g=0.0365, v=-0.3942$, and $N=7$. (c) Optical spectrum of the stable pattern state. (d) Differential phase evolution of three comb modes indicated by colored shading in (c). The differential phase is calculated as the derivative of the absolute phase $\varphi_{\mathrm{n}}$ over the temporal evolution, and it is indicative of the stability intrinsic to the repetition rate of the comb. 

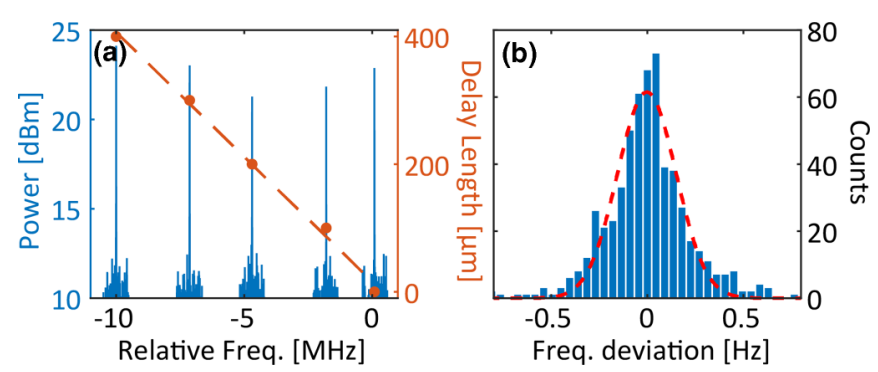

FIG. 8. (a) The average beat note frequencies between two selected microcomb lines and their adjacent reference frequency comb teeth (recorded by an oscilloscope) as a function of the decreasing main-cavity length. The raw frequency measurement, in megahertz, relative to the frequency for $0-\mu \mathrm{m}$ delay, is shown in blue. (b) Distribution of the microcomb output spectrum stemming from an equidistant mode spacing, including a Gaussian fit (dashed red line). Here, we consider the $-300-\mu$ m delay case with a frequency counter gate time of $100 \mathrm{~ms}$.

shown in Fig. 8(a) for the cases where the differential length of the delay line varies from 0 to $-400 \mu \mathrm{m}$. The resulting average change in repetition rate is plotted for the various cavity delay lengths with the raw frequency data displayed in blue. The linear increase in repetition rate by up to $10 \mathrm{MHz}$ as the fiber cavity length is decreased is clearly visible. The quality of the phase locking for these Turing pattern states can be then evaluated. A typical distribution of the frequency deviation stemming from an equidistant mode spacing for a gate time of $100 \mathrm{~ms}$ is shown in Fig. 8(b). This measurement was obtained by setting the reference comb repetition rate as a noninteger submultiple of the microcomb repetition rate. As such, the beating signals [indicated as B1-B2 and B2-B3 in Fig. 4(c)] were centered around a frequency of $18 \mathrm{MHz}$, which was passband filtered. We collected the ratio between the two signals in order to extract the stability of the repetition rate. Since our measurement system was not further phase locked, we observed slips of the measurements towards other beat notes, which have been removed from the dataset by setting a threshold at $\pm 5 \mathrm{~Hz}$.

The mean and the standard deviation of the frequency distributions in Fig. 8(a) is presented in Table I.

Our mean values are consistently in the same order of magnitude of the standard error, which are all in the range of $10 \mathrm{mHz}$, indicating that, even with short-time gates of $100 \mathrm{~ms}$, we can claim a stability better than $7 \times 10^{-14}$ for the $150-\mathrm{GHz}$ state. Our electronics unlocked several times during the measurement of the case at $-200 \mu \mathrm{m}$, which has a low number of counts and, for this reason, the highest standard error.

Typical values for the stability of the repetition rate associated to the free-running microcomb sources range from $10^{-9}$ to $10^{-12}$ for soliton states measured with these level of time gates [63,64]. Free-running Turing pattern-based sources, which are known for having a stronger phase lock, have been demonstrated in the same order of magnitude $\left(7 \times 10^{-14}\right)$ as our measurement but obtained with time gates of $1 \mathrm{~s}$ [40]. This shows the remarkable performances of our system, which can achieve the same level of stability averaging on gate times shorter by one order of magnitude. We believe that this enhanced stability is due to the intrinsic feedback that our nested cavities design allows. In addition, we maintain this excellent stability continuously over a tuning range of 10 $\mathrm{MHz}$, whereas designs, such as the one proposed in Ref. [40], require fixing the repetition rate to a specific value. Our tuning capabilities are in agreement with the theoretical calculation in Fig. 7(d), which shows the strong locking of the Turing pattern state also when the group velocity is detuned.

In conclusion, we study the formation of nonlocalized Turing patterns in a system where a nonlinear high- $Q$ microresonator is nested into an active fiber loop. By using a comprehensive mean-field model, we develop a MI analysis which explains the nature of these waves, revealing the dynamics of their formation from noise and explaining the ease with which they are observed. We demonstrate that these waves possess strong phase locking with frequency deviations of the repetition rate frequency being below a hertz. Furthermore, we show that the repetition rate can be controlled by simply acting on the main-cavity length which affects the modulational instability spectrum whereas maintaining the pulse quality. In this regard, the experimental results demonstrate that the repetition rate of these waves can be controlled over both fine (megahertz) and large (gigahertz) scales, with a continuous tuning of up to $10 \mathrm{MHz}$ verified experimentally. From a theoretical point of view, this paper will help increasing our understanding of nonlinear processes in multi cavity systems. Our results provide a pathway for designing practical microcomb devices that can be easily initiated and tuned by the end user, a fundamental requirement for the widespread use of these devices outside the laboratory environment.

\section{ACKNOWLEDGMENTS}

We thank G.-L. Oppo and B. Wetzel for insightful discussions. We acknowledge the support of the EPSRC Industrial Innovation Fellowship Programme under Grant No. EP/S001018/1 from INNOVATE UK, Project "IOTA" Grant

TABLE I. Results of the frequency uniformity measurements of data in Figs. 6 and 8 for time gates of $100 \mathrm{~ms}$.

\begin{tabular}{lcccc}
\hline \hline Relative delay $(\mu \mathrm{m})$ & Mean $(\mathrm{mHz})$ & Standard deviation $(\mathrm{mHz})$ & Approved counts & Relative deviation \\
\hline-400 & $-5.5 \pm 8.3$ & 213 & 655 & $5.5 \times 10^{-14}$ \\
-300 & $-10 \pm 11$ & 219 & 432 & 74 \\
-200 & $-12 \pm 20$ & 172 & 580 & $1.3 \times 10^{-13}$ \\
-100 & $-3.5 \pm 9.5$ & 228 & 588 & $6.3 \times 10^{-14}$ \\
0 & $-8.1 \pm 9.7$ & 235 & $6.4 \times 10^{-14}$ \\
\hline \hline
\end{tabular}


Agreement No. EP/R043566/1, and the University of Sussex RDF Programme. This project has received funding from the European Research Council (ERC) under the European Union's Horizon 2020 Research and Innovation Programme Grant Agreement No. 851758 (TELSCOMBE). M.R. acknowledges the support of the EPSRC through the Studentship No. EP/N509784/1. J.S.T.G. acknowledges funding from the Helena Normanton Fellowship of the University of Sussex. S.T.C. acknowledges support from the Research Grant Council of Hong Kong (Grant GRF No. 9042663). B.E.L. acknowledges support from the Strategic Priority Research Program of the Chinese Academy of Sciences (Grant
No. XDB24030300). R.M. is affiliated with the Institute of Fundamental and Frontier Sciences, University of Electronic Science and Technology of China, Chengdu, China and ITMO University, St. Petersburg, 199034, Russia as an adjoint faculty and acknowledges funding by the Natural Sciences and Engineering Research Council of Canada (NSERC) through the Strategic Grants Scheme, by the MESI PSR-SIIRI Initiative in Quebec, by the Canada Research Chair Program, as well as additional support by the Government of the Russian Federation through the ITMO Fellowship and Professorship Program (Grant No. 074-U 01) and by the 1000 Talents Sichuan Program.
[1] L. Lugiato, F. Prati, and M. Brambilla, Nonlinear Optical Systems (Cambridge University Press, Cambridge, UK, 2015).

[2] A. M. Turing, The chemical basis of morphogenesis, Philos. Trans. R. Soc. London, Ser. B 237, 37 (1952).

[3] L. A. Lugiato and R. Lefever, Spatial Dissipative Structures in Passive Optical Systems, Phys. Rev. Lett. 58, 2209 (1987).

[4] K. Staliūnas and V. J. Sánchez-Morcillo, Transverse Patterns in Nonlinear Optical Resonators (Springer, Berlin/Heidelberg, 2003).

[5] A. J. Scroggie, W. J. Firth, G. S. McDonald, M. Tlidi, R. Lefever, and L. A. Lugiato, Pattern formation in a passive Kerr cavity, Chaos, Solitons Fractals 4, 1323 (1994).

[6] T. Ackemann, Yu. A. Logvin, A. Heuer, and W. Lange, Transition between Positive and Negative Hexagons in Optical Pattern Formation, Phys. Rev. Lett. 75, 3450 (1995).

[7] G. D’Alessandro, E. Pampaloni, P.- L. Ramazza, S. Residori, and F. T. Arecchi, Amplitude equations and pattern formation in a liquid-crystal light-valve experiment, Phys. Rev. A 52, 4176 (1995).

[8] G.-L. Oppo, M. Brambilla, and L. A. Lugiato, Formation and evolution of roll patterns in optical parametric oscillators, Phys. Rev. A 49, 2028 (1994).

[9] A. M. Perego, N. Tarasov, D. V. Churkin, S. K. Turitsyn, and K. Staliunas, Pattern Generation by Dissipative Parametric Instability, Phys. Rev. Lett. 116, 028701 (2016).

[10] M. Haelterman, S. Trillo, and S. Wabnitz, Dissipative modulation instability in a nonlinear dispersive ring cavity, Opt. Commun. 91, 401 (1992).

[11] F. Leo, S. Coen, P. Kockaert, S.-P. Gorza, P. Emplit, and M. Haelterman, Temporal cavity solitons in one-dimensional Kerr media as bits in an all-optical buffer, Nat. Photonics 4, 471 (2010).

[12] M. Marconi, J. Javaloyes, S. Balle, and M. Giudici, How Lasing Localized Structures Evolve out of Passive Mode Locking, Phys. Rev. Lett. 112, 223901 (2014).

[13] A. Coillet, I. Balakireva, R. Henriet, K. Saleh, L. Larger, J. M. Dudley, C. R. Menyuk, and Y. K. Chembo, Azimuthal turing patterns, bright and dark cavity solitons in kerr combs generated with whispering-gallery-mode resonators, IEEE Photonics J. 5, 6100409 (2013)

[14] M. Anderson, Y. Wang, F. Leo, S. Coen, M. Erkintalo, and S. G. Murdoch, Coexistence of Multiple Nonlinear States in a Tristable Passive Kerr Resonator, Phys. Rev. X 7, 031031 (2017).
[15] A. U. Nielsen, B. Garbin, S. Coen, S. G. Murdoch, and M. Erkintalo, Coexistence and Interactions between Nonlinear States with Different Polarizations in a Monochromatically Driven Passive Kerr Resonator, Phys. Rev. Lett. 123, 013902 (2019).

[16] T. J. Kippenberg, R. Holzwarth, and S. A. Diddams, Microresonator-based optical frequency combs, Science 332, 555 (2011).

[17] A. Pasquazi et al., Micro-combs: A novel generation of optical sources, Phys. Rep. 729, 1 (2018).

[18] A. A. Savchenkov, A. B. Matsko, and L. Maleki, On Frequency Combs in Monolithic Resonators, Nanophotonics 5, 363 (2016).

[19] P. Grelu, Nonlinear Optical Cavity Dynamics: From Microresonators to Fiber Lasers (Wiley-VCH, Weinheim, Germany, 2016).

[20] T. J. Kippenberg, A. L. Gaeta, M. Lipson, and M. L. Gorodetsky, Dissipative Kerr solitons in optical microresonators, Science 361, eaan8083 (2018).

[21] A. L. Gaeta, M. Lipson, and T. J. Kippenberg, Photonic-chipbased frequency combs, Nat. Photonics 13, 158 (2019).

[22] B. Stern, X. Ji, Y. Okawachi, A. L. Gaeta, and M. Lipson, Battery-operated integrated frequency comb generator, Nature (London) 562, 401 (2018).

[23] E. Obrzud, S. Lecomte, and T. Herr, Temporal solitons in microresonators driven by optical pulses, Nat. Photonics 11, 600 (2017)

[24] W. Liang, A. A. Savchenkov, Z. Xie, J. F. McMillan, J. Burkhart, V. S. Ilchenko, C. W. Wong, A. B. Matsko, and L. Maleki, Miniature multioctave light source based on a monolithic microcavity, Optica 2, 40 (2015).

[25] L. Maleki, V. S. Ilchenko, and A. B. Matsko, Practical Applications of Microresonators in Optics and Photonics (CRC, Boca Raton, FL, 2018).

[26] S. Zhang, J. M. Silver, L. D. Bino, F. Copie, M. T. M. Woodley, G. N. Ghalanos, A. Ø. Svela, N. Moroney, and P. Del'Haye, Sub-milliwatt-level microresonator solitons with extended access range using an auxiliary laser, Optica 6, 206 (2019).

[27] L. A. Lugiato, F. Prati, M. L. Gorodetsky, and T. J. Kippenberg, From the Lugiato-Lefever equation to microresonator-based soliton Kerr frequency combs, Philos. Trans. R. Soc,. A 376, 20180113 (2018).

[28] S. Coen, H. G. Randle, T. Sylvestre, and M. Erkintalo, Modeling of octave-spanning Kerr frequency combs using a 
generalized mean-field Lugiato-Lefever model, Opt. Lett. 38, 37 (2013).

[29] M.-G. Suh, Q.-F. Yang, K. Y. Yang, X. Yi, and K. J. Vahala, Microresonator soliton dual-comb spectroscopy, Science 354, 600 (2016).

[30] P. Marin-Palomo, J. N. Kemal, M. Karpov, A. Kordts, J. Pfeifle, M. H. P. Pfeiffer, P. Trocha, S. Wolf, V. Brasch, M. H. Anderson, R. Rosenberger, K. Vijayan, W. Freude, T. J. Kippenberg, and C. Koos, Microresonator-based solitons for massively parallel coherent optical communications, Nature (London) 546, 274 (2017).

[31] M.-G. Suh and K. J. Vahala, Soliton microcomb range measurement, Science 359, 884 (2018).

[32] V. Brasch, M. Geiselmann, T. Herr, G. Lihachev, M. H. Pfeiffer, M. L. Gorodetsky, and T. J. Kippenberg, Photonic chip-based optical frequency comb using soliton Cherenkov radiation, Science 351, 357 (2016).

[33] D. T. Spencer et al., An optical-frequency synthesizer using integrated photonics, Nature (London) 557, 81 (2018).

[34] W. Liang, D. Eliyahu, V. S. Ilchenko, A. A. Savchenkov, A. B. Matsko, D. Seidel, and L. Maleki, High spectral purity Kerr frequency comb radio frequency photonic oscillator, Nat. Commun. 6, 7957 (2015).

[35] S.-W. Huang, J. Yang, M. Yu, B. H. McGuyer, D.-L. Kwong, T. Zelevinsky, and C. W. Wong, A broadband chip-scale optical frequency synthesizer at $2.7 \times 10^{-16}$ relative uncertainty, Sci. Adv. 2, e1501489 (2016).

[36] M. Yu, Y. Okawachi, A. G. Griffith, N. Picqué, M. Lipson, and A. L. Gaeta, Silicon-chip-based mid-infrared dual-comb spectroscopy, Nat. Commun. 9, 1869 (2018).

[37] A. Dutt, C. Joshi, X. Ji, J. Cardenas, Y. Okawachi, K. Luke, A. L. Gaeta, and M. Lipson, On-chip dual-comb source for spectroscopy, Sci. Adv. 4, e1701858 (2018).

[38] J. Pfeifle et al., Optimally Coherent Kerr Combs Generated with Crystalline Whispering Gallery Mode Resonators for Ultrahigh Capacity Fiber Communications, Phys. Rev. Lett. 114, 093902 (2015).

[39] A. Coillet and Y. Chembo, On the robustness of phase locking in Kerr optical frequency combs, Opt. Lett. 39, 1529 (2014).

[40] S.-W. Huang, J. Yang, S.-H. Yang, M. Yu, D.-L. Kwong, T. Zelevinsky, M. Jarrahi, and C. W. Wong, Globally Stable Microresonator Turing Pattern Formation for Coherent High-Power THz Radiation On-Chip, Phys. Rev. X 7, 041002 (2017).

[41] S. Diallo and Y. K. Chembo, Optimization of primary Kerr optical frequency combs for tunable microwave generation, Opt. Lett. 42, 3522 (2017).

[42] C. Godey, I. V. Balakireva, A. Coillet, and Y. K. Chembo, Stability analysis of the spatiotemporal Lugiato-Lefever model for Kerr optical frequency combs in the anomalous and normal dispersion regimes, Phys. Rev. A 89, 063814 (2014).

[43] S. Coulibaly, M. Taki, A. Bendahmane, G. Millot, B. Kibler, and M. G. Clerc, Turbulence-Induced Rogue Waves in Kerr Resonators, Phys. Rev. X 9, 011054 (2019).

[44] M. Peccianti, A. Pasquazi, Y. Park, B. E. Little, S. T. Chu, D. J. Moss, and R. Morandotti, Demonstration of a stable ultrafast laser based on a nonlinear microcavity, Nat. Commun. 3, 765 (2012).

[45] A. Pasquazi, M. Peccianti, B. E. Little, S. T. Chu, D. J. Moss, and R. Morandotti, Stable, dual mode, high repetition rate mode-locked laser based on a microring resonator, Opt. Express 20, 27355 (2012).

[46] H. Bao, A. Cooper, S. T. Chu, D. J. Moss, R. Morandotti, B. E. Little, M. Peccianti, and A. Pasquazi, Type-II micro-comb generation in a filter-driven four wave mixing laser [Invited], Photonics Res. 6, B67 (2018).

[47] H. Bao et al., Laser cavity-soliton microcombs, Nat. Photonics 13, 384 (2019).

[48] W. Wang et al., Repetition Rate Multiplication Pulsed Laser Source Based on a Microring Resonator, ACS Photonics 4, 1677 (2017).

[49] Y. K. Chembo and C. R. Menyuk, Spatiotemporal LugiatoLefever formalism for Kerr-comb generation in whisperinggallery-mode resonators, Phys. Rev. A 87, 053852 (2013).

[50] H. A. Haus and W. Huang, and Coupled-mode theory, Proc. IEEE 79, 1505 (1991).

[51] B. A. Malomed, Solitary pulses in linearly coupled GinzburgLandau equations, Chaos 17, 037117 (2007).

[52] J. Atai and B. A. Malomed, Stability and interactions of solitons in two-component active systems, Phys. Rev. E 54, 4371 (1996).

[53] A. Sigler, B. A. Malomed, and D. V. Skryabin, Localized states in a triangular set of linearly coupled complex GinzburgLandau equations, Phys. Rev. E 74, 066604 (2006).

[54] A. J. Scroggie, W. J. Firth, and G.-L. Oppo, Cavity-soliton laser with frequency-selective feedback, Phys. Rev. A 80, 013829 (2009).

[55] A. Haboucha, H. Leblond, M. Salhi, A. Komarov, and F. Sanchez, Analysis of soliton pattern formation in passively mode-locked fiber lasers, Phys. Rev. A 78, 043806 (2008).

[56] R. Si Fodil, F. Amrani, C. Yang, A. Kellou, and Ph. Grelu, Adjustable high-repetition-rate pulse trains in a passively-modelocked fiber laser, Phys. Rev. A 94, 013813 (2016).

[57] N. Tarasov, A. M. Perego, D. V. Churkin, K. Staliunas, and S. K. Turitsyn, Mode-locking via dissipative Faraday instability, Nat. Commun. 7, 12441 (2016).

[58] F. Copie, M. Conforti, A. Kudlinski, A. Mussot, and S. Trillo, Competing Turing and Faraday Instabilities in Longitudinally Modulated Passive Resonators, Phys. Rev. Lett. 116, 143901 (2016).

[59] D. J. Moss, R. Morandotti, A. L. Gaeta, and M. Lipson, New CMOS-compatible platforms based on silicon nitride and Hydex for nonlinear optics, Nat. Photonics 7, 597 (2013).

[60] P. Del'Haye, O. Arcizet, M. L. Gorodetsky, R. Holzwarth, and T. J. Kippenberg, Frequency comb assisted diode laser spectroscopy for measurement of microcavity dispersion, Nat. Photonics 3, 529 (2009).

[61] Th. Udem, J. Reichert, R. Holzwarth, and T. W. Hänsch, Accurate measurement of large optical frequency differences with a mode-locked laser, Opt. Lett. 24, 881 (1999).

[62] P. Del'Haye, A. Schliesser, O. Arcizet, T. Wilken, R. Holzwarth, and T. J. Kippenberg, Optical frequency comb generation from a monolithic microresonator, Nature (London) 450, 1214 (2007).

[63] S. Zhang, J. M. Silver, X. Shang, L. Del Bino, N. M. Ridler, and P. Del'Haye, Terahertz wave generation using a soliton microcomb, Opt. Express 27, 35257 (2019).

[64] W. Weng, E. Lucas, G. Lihachev, V. E. Lobanov, H. Guo, M. L. Gorodetsky, and T. J. Kippenberg, Spectral Purification of Microwave Signals with Disciplined Dissipative Kerr Solitons, Phys. Rev. Lett. 122, 013902 (2019). 